Review began 09/22/2021 Review ended 10/18/2021 Published 10/19/2021

(c) Copyright 2021

Ali et al. This is an open access article distributed under the terms of the Creative Commons Attribution License CC-BY 4.0., which permits unrestricted use, distribution, and reproduction in any medium, provided the original author and source are credited.

\section{Acute Myocardial Injury and Rhabdomyolysis in COVID-19 Patients: Incidence and Mortality}

Liaquat Ali ${ }^{1,2}$, Imran Mohammed ${ }^{3}$, Imran Janjua ${ }^{3}$, Muhammad Naeem ${ }^{3}$, Gholam Adeli ${ }^{1}$, Osama Elalamy ${ }^{1}$, Mohammad Alhatou ${ }^{1}$, Naveed Akhtar ${ }^{1}$, Beatriz Canibano ${ }^{1}$, Ambreen Iqrar ${ }^{1}$

1. Neurology, Hamad General Hospital, Doha, QAT 2. Neurology, Weill Cornell Medicine, Doha, QAT 3. Internal Medicine, Hamad General Hospital, Doha, QAT

Corresponding author: Liaquat Ali, lali5@hamad.qa

\section{Abstract}

\section{Background}

Myocardial injury has been defined as an elevated troponin level. The frequency of acute myocardial injury of hospitalized coronavirus disease 2019 (COVID-19) patients ranges from 7\% to 36\%. COVID-19 patients with cardiovascular disease (CVD) have a four-fold higher risk of mortality (odds ratio, 4.33; CI 95\%, 3.165.94). In COVID-19 hospitalized patients' study showed mortality rate was $18.5 \%$. Rhabdomyolysis is considered as muscle necrosis and the release of intracellular muscles elements and enzymes into blood. In one of retrospective cohort study of COVID-19 hospitalized patients, incidence of rhabdomyolysis was $16.7 \%$.

\section{Materials and methods}

This retrospective observational study consisted of 413 COVID-19 hospitalized patients. Patients with rhabdomyolysis was defined as creatine kinase level greater than 1,000 U/L and acute myocardial injury was defined as serum high-sensitivity troponin-T for males greater than $30 \mathrm{ng} / \mathrm{l}$ and for female greater than 20 ng/l. The primary outcome was in-hospital mortality of COVID-19 patients with acute myocardial injury and rhabdomyolysis.

\section{Results}

The incidence of acute myocardial injury and rhabdomyolysis in hospitalized COVID-19 patients was $23.9 \%$ (99) and $15.7 \%$ (65), respectively. The mortality rate of in hospitalized COVID-19 patients who developed acute myocardial injury (28.3\%) was significantly higher in comparison to patients who developed rhabdomyolysis (13.8\%).

\section{Discussion}

The binding of SARS-CoV-2 virus to the angiotensin-converting enzyme 2 (ACE2) is a critical step in the pathophysiology in patients with COVID-19. There may be diverse direct and indirect mechanisms of acute myocardial injury in COVID-19 including ischemic injury, hypoxic injury (MI type 2), direct viral myocarditis, stress cardiomyopathy and systemic cytokine storm. Musculoskeletal injury may be caused by direct viral myositis or indirectly by host immune hyperinflammatory cytokine storm response that leads to skeletal muscle fiber proteolysis and fibrosis.

\section{Conclusions}

Acute myocardial injury and rhabdomyolysis were underreported in COVID-19 patients. The incidence and mortality of acute myocardial injury are higher than that of rhabdomyolysis in COVID-19 hospitalized patients. The outcome was worse in COVID-19 patients with severe acute myocardial injury. Patients with acute myocardial injury and rhabdomyolysis may get benefits from rehabilitation programs.

Categories: Cardiology, Internal Medicine, Neurology

Keywords: myocardial ischemia type 2(mi type 2), myocardial injury, rhabdomyolysis, creatine kinase (ck), angiotensin converting enzyme 2 (ace2), coronavirus disease 2019 (covid-19), severe acute respiratory syndrome coronavirus-2 (sars-cov-2)

\section{Introduction}

A novel human RNA coronavirus named as severe acute respiratory syndrome coronavirus-2 (SARS-CoV-2) was the cause of a cluster of pneumonia cases in Wuhan, China, in late 2019 and which was later called COVID-19 (coronavirus disease 2019) [1].

COVID-19 patients have cardiac manifestations including acute myocardial injury, heart failure, cardiogenic 
shock and arrhythmia. Acute myocardial injury is defined as an elevated troponin level and all conditions causing cardiomyocyte death [2]. The frequency of acute myocardial injury of COVID-19 hospitalized patients is variable from 7 to $36 \%$ due to the severity of COVID-19, use of troponin assays, $99^{\text {th }}$ percentile threshold and sampling times [3,4]. COVID-19 patients with cardiovascular disease (CVD) have a four-fold higher risk of mortality (odds ratio, 4.33; CI 95\%, 3.16 to 5.94) [5]. In a study of COVID-19 hospitalized patients from New York, USA, the mortality rate was 18.5\% [3]. COVID-19 may directly or indirectly affect the cardiovascular system causing acute coronary system (ACS), myocarditis and electrical heart disease.

Rhabdomyolysis is considered muscle necrosis and the release of intracellular muscles elements and enzymes into blood. Rhabdomyolysis is defined as creatine kinase (CK) level more than five times of the upper limit of normal (>1000 IU/L) [6]. In rhabdomyolysis, patients may present with muscles pain or weakness, red to brown color of urine (due to myoglobinuria) and markedly elevated creatine kinase (CK) and myoglobin levels. The other manifestations included electrolytes abnormalities ( such as hyperkalemia, hyperphosphatemia, hyperuricemia, hypocalcemia and metabolic acidosis) and later complications included acute kidney injury (AKI), compartment syndrome and rarely disseminated intravascular coagulation (DIC). Trauma, immobilization, sepsis, exertional heat stroke, generalized tonic colonic seizures, vascular and cardiac surgeries are the most common conditions associated with rhabdomyolysis. Rhabdomyolysis rarely occurs in association with an inflammatory myopathy. Rhabdomyolysis associated with COVID-19 has been reported in a few and some retrospective studies. In one of retrospective cohort study of 140 patients, the incidence of rhabdomyolysis in hospitalized COVID-19 patients was $16.7 \%$. The mortality rate was $47.1 \%$ in COVID-19 with rhabdomyolysis as in comparison to $26.4 \%$ in COVID-19 without rhabdomyolysis [7]. The objective of this study was to determine the incidence of acute myocardial injury and rhabdomyolysis in patients with COVID-19, its outcome and clinical implications.

\section{Materials And Methods}

This was a retrospective observational study at Hamad Medical Corporation (HMC), Doha, Qatar. They were allocated tertiary care multidisciplinary hospitals by the government to manage patients with COVID-19 infection. We obtained data of 413 with COVID-19 from 01 January 2020 to 31 December 2020. Patients were enrolled from hospital electronic medical records (EMR) by applying search criteria. We collected information of patients demographics (age, gender, BMI, nationality), presenting symptoms, comorbidities ( like hypertension, diabetes, obesity, smoking, ischemic heart disease, stroke and chronic kidney disease etc), inpatients laboratory values and electronic discharge summaries. The study was approved by the institutional review committee (MRC-01-20-523) at Hamad Medical Corporation (HMC), Doha, Qatar and patients informed consent were waived due to retrospective study and urgently required new data collection of COVID-19 pandemic, results for improves clinical practice. Inclusion criteria were adult age greater than 18 years and COVID-19 confirmation, which was defined based on the positive result of real-time reverse transcription-polymerase chain reaction (RT-PCR) on both nasopharyngeal and oropharyngeal swab specimens. Rhabdomyolysis was defined as when serum creatine kinase (CK) level greater than $1000 \mathrm{U} / \mathrm{L}$ and skeletal muscles injury was defined as when serum creatine kinase level greater than $300 \mathrm{U} / \mathrm{L}$ or serum myoglobin level greater than $72 \mathrm{ng} / \mathrm{ml}$ with or without myalgia, muscles weakness and cola-colored urine. Acute myocardial injury is defined as peak serum high-sensitivity troponin-T greater than $30 \mathrm{ng} / \mathrm{l}$ for males, and greater than $70 \mathrm{ng} / \mathrm{l}$ for females during hospitalization. We excluded patients who had evidence of secondary rhabdomyolysis due to traumatic or crush injury, heat-related stroke, Ischemic, exertional skeletal muscles injury, generalized tonic colonic seizures, alcohol abuse, certain medicines and toxic substances that were known to cause acquired myopathies and known inherited myopathies prior to admission. Primary outcome was in-hospital mortality. Routine laboratory testing (Complete blood count(CBC), complete metabolic panel (CMP), prothrombin time (PT)/activated partial prothrombin time (APPT)/ international normalized ratio (INR), urea, creatinine, electrolytes, liver function test (LFT), C reactive protein (CRP), Ferritin, lactate dehydrogenase (LDH), D-dimer, interleukine-6 (IL-6), lactic acid, creatine kinase (CK), myoglobin, troponin etc.), radiologic examination included chest $\mathrm{x}$ - rays and chest CT that was performed according to standard clinical practice and CDC Qatar guidelines. The primary objective was to determine the incidence of acute myocardial injury and rhabdomyolysis in COVID-19 hospitalized patients and the secondary objective was to determine the mortality in rhabdomyolysis and acute myocardial injury COVID-19 patients. The data were summarized by descriptive statistics; results were reported as mean, median, interquartile ranges (IQR) and standard deviations as appropriate. Categorical variables were expressed as counts and percentages. Continuous variables were compared by using unpaired Wilcox rank-sum test. Proportions for categorical variables were compared by using $\mathrm{X}^{2}$ test. The significant threshold was set at $\mathrm{P}<0.05$. All statistical analyses were done by using the statistical package SPSS 24.0 (SPSS Inc. Chicago, IL).

\section{Results}

Four hundred and thirteen (413) hospitalized patients with COVID-19 were recruited in this study. The patients demographics are shown in Table 1 and the clinical characteristics are shown in Table 2 . 


\section{Cureus}

\begin{tabular}{|c|c|c|}
\hline Age (years) & Frequency(n) & Percentage (\%) \\
\hline 22 to 54 (young) & 234 & $56.7 \%$ \\
\hline 55-74 (middle age) & 156 & $37.8 \%$ \\
\hline 75-84 (old age) & 21 & $5.1 \%$ \\
\hline >85 (older age) & 2 & $0.5 \%$ \\
\hline \multicolumn{3}{|l|}{ Gender } \\
\hline Male & 389 & $94 \%$ \\
\hline Female & 23 & 6.0 \\
\hline \multicolumn{3}{|l|}{ Nationality } \\
\hline Indian & 84 & $20.3 \%$ \\
\hline Bangladesh & 52 & 12.5 \\
\hline Qatari & 42 & 10.1 \\
\hline Nepalese & 38 & 9.2 \\
\hline Filipino & 36 & 8.7 \\
\hline Pakistani & 36 & 8.7 \\
\hline Egyptian & 29 & 7.0 \\
\hline
\end{tabular}

\section{TABLE 1: Demographics (age, gender, nationality).}

The median age was 52 years and the majority were young males ( $<54$ years) and of Indian nationality.

\begin{tabular}{|c|c|c|}
\hline Symptoms & Frequency (n) & Percentage $\%$ \\
\hline Fever & 321 & $77.5 \%$ \\
\hline Cough & 279 & 67.4 \\
\hline Shortness of breath (with hypoxia and required oxygen) & 243 & 58.7 \\
\hline Myalgia & 116 & $28 \%$ \\
\hline Headache & 43 & 10.4 \\
\hline Chest pain & 41 & 9.9 \\
\hline Vertigo & 24 & 5.8 \\
\hline Muscle tenderness and weakness & 16 & 3.9 \\
\hline Anosmia & 12 & 2.9 \\
\hline Dysuria & 6 & 1.4 \\
\hline Cola-color urine & 1 & 0.2 \\
\hline
\end{tabular}

\section{TABLE 2: Clinical manifestations.}

Clinical manifestations in COVID-19 included fever, cough, SOB, myalgia, headache, chest pain in descending order.

The median age was 52 years (range 22 to 86), the majority were male (94\%) and $57 \%$ were less than 55 years. The most common comorbid illnesses were hypertension (47.6\%), diabetes mellitus (46.9\%), obesity (21\%), chronic kidney disease (10\%), ischemic heart disease (9.7\%) and smoker (6.8\%) as shown in Table 3. 


\section{Cureus}

\begin{tabular}{|c|c|c|}
\hline Underlying risk factors & Frequency $(\mathbf{n})$ & Percentage (\%) \\
\hline Hypertension & 197 & $47.6 \%$ \\
\hline Diabetes & 194 & $46.9 \%$ \\
\hline Obesity & 88 & $21 \%$ \\
\hline Newly diagnosed DM (on admission $\mathrm{HbA} 1 \mathrm{c}>6.5 \%$ ) & 42 & $10 \%$ \\
\hline Chronic kidney disease & 43 & $10 \%$ \\
\hline Ischemic heart disease & 40 & $9.7 \%$ \\
\hline Smoker & 28 & $6.8 \%$ \\
\hline Asthma & 15 & $3.6 \%$ \\
\hline Malignancy & 12 & $2.91 \%$ \\
\hline
\end{tabular}

\section{TABLE 3: Common comorbid illnesses and risk factors for COVID-19.}

The most common medical comorbid conditions and risk factors for COVID-19 were included; hypertension, diabetes mellitus (DM), obesity, chronic kidney disease, ischemic heart disease and smoker in descending order.

Among all COVID-19 patients, other multiorgan dysfunction included; 37\% (153) were cytokine storm, $36.7 \%$ (152) were acute kidney injury (AKI), 63.07\% (41) were AKI in patients with rhabdomyolysis, 35.7\% (148) were skeletal muscle injury (as shown in bar chart Figure 1), 27.5\% (114) were acute liver injury, 23.9\% (99) were acute myocardial injury, $15.7 \%$ (65) were rhabdomyolysis and $11.4 \%$ (47) were acute heart failure as shown in Table 4. 45\% (187) COVID-19 patients were admitted in MICU, 29\% (120) were mechanically ventilated and $16 \%$ (67) were managed with noninvasive ventilation (NIV). $8.5 \%$ (35) MICU patients died with COVID-19 complications and multiorgan failure. The mortality rate of COVID-19 patients with acute myocardial injury, cytokine storm syndrome and rhabdomyolysis were $28.3 \%(28), 19.6 \%$ (30) and $13.8 \%$ (9) in descending order. There were significantly high inflammatory markers (ferritin, CRP, D-dimer and IL-6) in both rhabdomyolysis and acute myocardial injury. The most common ECG changes (23.2\%) were sinus tachycardia (5.8\%), T waves abnormalities (5.6\%), atrial fibrillation(3.6\%), asystole (3.6\%) in descending order as shown in Table 5 .

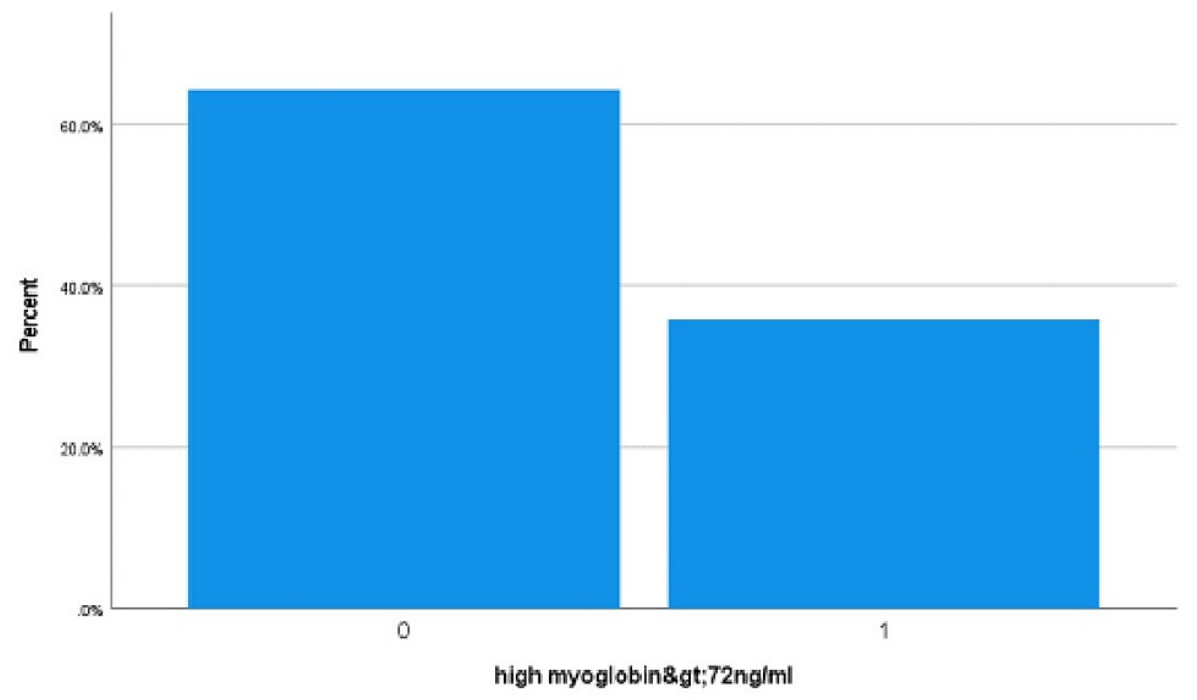

\section{FIGURE 1: Skeletal muscles injury (myoglobin).}

In COVID-19 hospitalized patients, there were $35.7 \%$ (148) high serum myoglobin (>72 $\mathrm{ng} / \mathrm{ml}$ ) as shown in the bar chart ( 1 represents greater than $72 \mathrm{ng} / \mathrm{ml}$ myoglobin level, zero represents less than $72 \mathrm{ng} / \mathrm{ml}$ ). 


\section{Cureus}

\section{Laboratory features}

Serum ferritin $>500 \mathrm{mcg} / \mathrm{L}$

Ferritin $>500 \mathrm{mcg} / \mathrm{L}$ in rhabdomyolysis (65)

Ferritin $>500 \mathrm{mcg} / \mathrm{L}$ in acute myocardial injury (99)

C-reactive protein levels (>100mg/l)

C-reactive protein levels (>100mg/l) in rhabdomyolysis (65)

C-reactive protein levels $(>100 \mathrm{mg} / \mathrm{l})$ in acute myocardial injury (99)

D-Dimer $>1000 \mathrm{ng} / \mathrm{ml}$

D-Dimer $>1000 \mathrm{ng} / \mathrm{ml}$ in rhabdomyolysis (65)

D-Dimer $>1000 \mathrm{ng} / \mathrm{ml}$ in acute myocardial injury (99)

IL6>70 pg/ml

IL6>70 pg/ml in Rhabdomyolysis (65)

IL6>70 pg/ml in acute myocardial injury (99)

Acute myocardial injury in patient high inflammatory marker (103) (ferritin, CRP, D dimer, IL-6)

Rhabdomyolysis in patient high inflammatory markers (103) (ferritin, CRP, D dimer, IL-6)

$\mathrm{LDH}>245$ units/L

Serum $\mathrm{CK}>300 \mathrm{u} / \mathrm{L}$

Serum myoglobin $>72 \mathrm{ng} / \mathrm{dl}$

Acute kidney injury (AKI) (urea $>8 \mathrm{mmol} / \mathrm{L}$, creatinine $>106 \mathrm{ml} / \mathrm{min}$ )

Acute kidney injury in rhabdomyolysis (65)

ALT or AST $>200 \mathrm{U} / \mathrm{L}$ (Acute liver injury)

Troponin-T hs (male>30, female $>20 n g /$ ) Acute myocardial injury

Procalcitonin (> 2ng/ml)

Serum CK>1000 U/L (Rhabdomyolysis)

Pro-BNP (>1800pg/ml) AHF

Lactic acid $>4 \mathrm{mml} / \mathrm{l}$

Echocardiography

Mild low ejection fraction (51-41\%)

Moderate low ejection fraction (40-30\%)

Severe low ejection fraction $(<30 \%)$

\begin{tabular}{|c|c|}
\hline Frequency $(n)$ & Percentage (\%) \\
\hline 295 & $71 \%$ \\
\hline 58 & $89.2 \%$ \\
\hline 86 & $86.8 \%$ \\
\hline 253 & $61 \%$ \\
\hline 57 & $87.8 \%$ \\
\hline 86 & $86.8 \%$ \\
\hline 223 & $53.9 \%$ \\
\hline 51 & $78.5 \%$ \\
\hline 74 & $74.7 \%$ \\
\hline 153 & $37 \%$ \\
\hline 42 & $64.6 \%$ \\
\hline 67 & $67.7 \%$ \\
\hline 53 & $51.45 \%$ \\
\hline 29 & $28.15 \%$ \\
\hline 274 & $66.2 \%$ \\
\hline 163 & $39.4 \%$ \\
\hline 148 & $35.7 \%$ \\
\hline 152 & $36.7 \%$ \\
\hline 41 & $63.07 \%$ \\
\hline 114 & $27.5 \%$ \\
\hline 99 & $23.9 \%$ \\
\hline 90 & 22.2 \\
\hline 65 & $15.7 \%$ \\
\hline 47 & 11.4 \\
\hline 26 & 6.3 \\
\hline 32 & 7.7 \\
\hline 11 & 2.7 \\
\hline 7 & 1.7 \\
\hline
\end{tabular}

\section{TABLE 4: Laboratory and echocardiographic findings.}

Laboratory features associated with severe COVID-19 infection included $71 \%$ were high ferritin level, $66.2 \%$ were high LDH, $61 \%$ were high CRP level, $53.9 \%$ were high D-dimer, 39.4\% were high CK level, 35.7\% were high myoglobin level, $22.2 \%$ were high procalcitonin, $15.7 \%$ were rhabdomyolysis and $6.3 \%$ were high lactic acid. $7.7 \%$ hospitalized patients were performed Echocardiography showed $3.4 \%$ were mild low EF, $1.7 \%$ were severe low EF and $0.5 \%$ were moderate low $E F$. 


\section{Cureus}

\begin{tabular}{|c|c|c|}
\hline ECG & Frequency(n) & Percentage (\%) \\
\hline ECG changes (413) & 96 & $23.2 \%$ \\
\hline Sinus Tachycardia & 24 & $5.8 \%$ \\
\hline T waves changes & 23 & $5.6 \%$ \\
\hline Asystole & 15 & $3.6 \%$ \\
\hline Atrial Fibrillation & 15 & $3.6 \%$ \\
\hline AV block abnormalities & 10 & $2.4 \%$ \\
\hline Sinus bradycardia & 9 & $2.2 \%$ \\
\hline ST elevation changes & 6 & $1.4 \%$ \\
\hline Prolonged QTc interval & 6 & $1.4 \%$ \\
\hline Pulseless electrical activity (PEA) & 5 & $1.2 \%$ \\
\hline Ventricular tachycardia (VT) & 2 & $0.5 \%$ \\
\hline Ventricular Fibrillation (VF) & 2 & $0.5 \%$ \\
\hline
\end{tabular}

TABLE 5: ECG changes (413 patients).

The most common ECG changes (23.2\%) were sinus tachycardia (5.8\%), T waves abnormalities (5.6\%), atrial fibrillation(3.6\%), asystole (3.6\%) in descending order.

During the study period of 413 enrolled patients, 89.6\% (370) were discharged home and $8.5 \%$ (35) died in hospital, and $1.9 \%$ (8) were shifted to a rehabilitation center for physical therapy. Among all COVID-19 hospitalized patients with high inflammatory markers (103), 51.45\% (53) were acute myocardial injury and 28.15\% (29) were rhabdomyolysis as shown in Table 4. There was a significantly higher acute myocardial injury in comparison with rhabdomyolysis in patients with high inflammatory markers (51.45\% Vs $28.15 \%$ ). Among all COVID-19 hospitalized patients, 10.6\% (44) were both acute myocardial injury and heart failure, $6.8 \%$ (28) were both acute myocardial injury and rhabdomyolysis. The incidence of acute myocardial injury was $23.9 \%$ (99) and the incidence of rhabdomyolysis was $15.7 \%$ (65) in all COVID-19 hospitalized patients. The mortality rate of COVID-19 hospitalized patients who developed acute myocardial injury was significantly higher in comparison with patients who developed rhabdomyolysis (28.3\% vs. $13.8 \%)$.

\section{Discussion}

The purpose of this study was to investigate the incidence and mortality of acute myocardial injury and rhabdomyolysis in COVID-19 hospitalized patients in designated COVID-19 treatment centers in Qatar. The most important step in the pathogenesis of COVID-19 is the binding of SARS-CoV-2 to angiotensinconverting enzyme 2 (ACE 2) receptors [8]. ACE-2 has a wide distribution in multiple organs including skeletal and cardiac muscles [9]. Cytokine storm is defined as an activation cascade of auto-amplifying cytokine production due to the unregulated host innate and adaptive immune response activated by SARSCoV-2 infection. There is increasing evidence that cytokines such as IL-6, IL-1 beta, IFN-gamma and TNFalpha play important role in pathogenesis of cytokine storms in COVID-19. Patients may acutely deteriorate in cytokine storms and develop acute respiratory distress syndrome (ARDS) and other multiorgan failure (MOF) [10-12].

SARS-CoV-2 related viral myositis may be attributable to direct myocyte invasion or induction of autoimmunity. In one of the autopsy series of patients who had died with COVID-19, concluded that most individuals with COVID-19 showed signs of myositis ranging from mild to severe and associated with duration of illness. Inflammation of skeletal muscles was more distinct than cardiac inflammation. Detection of viral load was low or negative in most skeletal and cardiac muscles and probably attributable to circulating viral RNA rather than genuine infection of myocytes. This suggests that SARS-CoV-2 may be associated with a post-infectious, immune-mediated myopathy [13]. There may be various direct and indirect mechanisms of acute myocardial injury in COVID-19 including ischemic injury, hypoxic injury (MI type 2), direct viral myocarditis, stress cardiomyopathy and systemic cytokine storm.

There may be various direct and indirect mechanisms of acute myocardial injury in COVID-19 including ischemic injury caused by cardiac microvascular damage or endotheliitis or epicardial coronary artery disease as thrombotic and plaque rupture or thrombosis due to hypercoagulability, hypoxic injury (supply- 
demand mismatch as myocardial ischemia type 2), direct viral myocarditis, stress cardiomyopathy and systemic cytokine storm $[14,15]$. The role of ACE-2 receptor-related signaling pathways dysfunction in COVID-19-related cardiac injury is unknown.

The exact mechanism of skeletal muscle damage with SARS-CoV-2 viral infections is not fully understood. It is unclear if SARS-CoV-2 infects muscles directly [7]. Musculoskeletal injury may be caused by indirect host cytokine storm immune response, leading to skeletal muscle fiber proteolysis and fibrosis [16]. Among all COVID-19 hospitalized patients, 10.6\% (44) were both acute myocardial injury and heart failure, 6.8\% (28) were both acute myocardial injury and rhabdomyolysis, 36.7\% (152) were acute kidney injury (AKI) and $63.07 \%$ (41) were AKI in rhabdomyolysis patients. There was a significantly higher acute myocardial injury in comparison with rhabdomyolysis in patients with high inflammatory markers (51.45\% vs. $28.15 \%)$. In this study, the incidence of acute myocardial injury was $23.9 \%$ and the incidence of rhabdomyolysis in COVID19 patients were $15.7 \%$. The mortality rate of acute myocardial injury (28.3\%) was significantly higher in comparison with rhabdomyolysis (13.8\%) in COVID-19 patients.

\section{Conclusions}

Acute myocardial injury and rhabdomyolysis are important complications that are underreported among COVID-19 hospitalized patients. Patients with COVID-19 may present with a broad spectrum of cardiac manifestations as no clinical evidence of heart disease, asymptomatic heart disease (have cardiac test abnormalities) and symptomatic heart disease. The common neuromuscular manifestation of COVID-19 patients are myalgias, skeletal muscles injury and rhabdomyolysis. There was a significantly higher acute myocardial injury in comparison with rhabdomyolysis among COVID-19 patients with high inflammatory markers. The incidence and mortality of acute myocardial injury in comparison with rhabdomyolysis were significantly higher among COVID-19 patients. In future, more prospective studies will be required with a focus on short- and long-term outcomes of COVID-19 patients and to get the benefit of using rehabilitation programs.

\section{Additional Information \\ Disclosures}

Human subjects: Consent was obtained or waived by all participants in this study. Medical Research Centre issued approval MRC 01-20-523. Approval for this study granted by the institutional review board (MRC 0120-523) at Hamad Medical Corporation (HMC), Doha, Qatar. . Animal subjects: All authors have confirmed that this study did not involve animal subjects or tissue. Conflicts of interest: In compliance with the ICMJE uniform disclosure form, all authors declare the following: Payment/services info: All authors have declared that no financial support was received from any organization for the submitted work. Financial relationships: All authors have declared that they have no financial relationships at present or within the previous three years with any organizations that might have an interest in the submitted work. Other relationships: All authors have declared that there are no other relationships or activities that could appear to have influenced the submitted work.

\section{Acknowledgements}

Adnan Khan, Mary Metreenj, Ansaf Ahmed, Jessy Jacob, Lucy Karugu, Neelima John, Kazi Muntaha, Mirza Baig.

\section{References}

1. She J, Jiang J, Ye L, Hu L, Bai C, Song Y: 2019 novel coronavirus of pneumonia in Wuhan, China: emerging attack and management strategies. Clin Transl Med. 2020, 9:19. 10.1186/s40169-020-00271-Z

2. Sandoval Y, Januzzi JL Jr, Jaffe AS: Cardiac troponin for assessment of myocardial injury in COVID-19: JACC review topic of the week. J Am Coll Cardiol. 2020, 76:1244-58. 10.1016/j.jacc.2020.06.068

3. Lala A, Johnson KW, Januzzi JL, et al.: Prevalence and impact of myocardial injury in patients hospitalized with COVID-19 infection. J Am Coll Cardiol. 2020, 76:533-46. 10.1016/j.jacc.2020.06.007

4. Sandoval Y, Bielinski SJ, Daniels LB, et al.: Atherosclerotic cardiovascular disease risk stratification based on measurements of troponin and coronary artery calcium. J Am Coll Cardiol. 2020, 76:357-70. 10.1016/j.jacc.2020.05.057

5. Shi S, Qin M, Shen B, et al.: Association of cardiac injury with mortality in hospitalized patients with COVID-19 in Wuhan, China. JAMA Cardiol. 2020, 5:802-10. 10.1001/jamacardio.2020.0950

6. Melli G, Chaudhry V, Cornblath DR: Rhabdomyolysis: an evaluation of 475 hospitalized patients . Medicine. 2005, 84:377-85. 10.1097/01.md.0000188565.48918.41

7. Haroun MW, Dieiev V, Kang J, et al.: Rhabdomyolysis in COVID-19 patients: a retrospective observational study. Cureus. 2021, 13:e12552. 10.7759/cureus.12552

8. Ang CW, Jacobs BC, Laman JD: The Guillain-Barré syndrome: a true case of molecular mimicry . Trends Immunol. 2004, 25:61-6. 10.1016/j.it.2003.12.004

9. Crum-Cianflone NF: Bacterial, fungal, parasitic, and viral myositis. Clin Microbiol Rev. 2008, 21:473-94. 10.1128/CMR.00001-08

10. Cron R, Behrens EM: Cytokine Storm Syndrome. Springer Nature Switzerland AG (ed): Springer International Publishing, Cham; 2019. 10.1007/978-3-030-22094-5 


\section{Cureus}

11. Mehta P, McAuley DF, Brown M, Sanchez E, Tattersall RS, Manson JJ: COVID-19: consider cytokine storm syndromes and immunosuppression. Lancet. 2020, 395:1033-4. 10.1016/S0140-6736(20)30628-0

12. Chen R, Lan Z, Ye J, et al.: Cytokine storm: the primary determinant for the pathophysiological evolution of COVID-19 deterioration. Front Immunol. 2021, 12:589095. 10.3389/fimmu.2021.589095

13. Aschman T, Schneider J, Greuel S, et al.: Association between SARS-CoV-2 infection and immune-mediated myopathy in patients who have died. JAMA Neurol. 2021, 78:948-60. 10.1001/jamaneurol.2021.2004

14. Fox SE, Lameira FS, Rinker EB, Vander Heide RS: Cardiac endotheliitis and multisystem inflammatory syndrome after COVID-19. Ann Intern Med. 2020, 173:1025-7. 10.7326/L20-0882

15. Libby P, Lüscher T: COVID-19 is, in the end, an endothelial disease . Eur Heart J. 2020, 41:3038-44. 10.1093/eurhearti/ehaa623

16. Disser NP, De Micheli AJ, Schonk MM, et al.: Musculoskeletal Consequences of COVID-19. J Bone Joint Surg Am. 2020, 102:1197-204. 10.2106/JBIS.20.00847 